\title{
Explicit Expression for a First Integral for a Class of Two-dimensional Differential System
}

\author{
Rachid Boukoucha ${ }^{* 1}$, Mouna Yahiaoui ${ }^{2}$ \\ 1,2 Lab. de Mathématiques Appliquées, Université de Bejaia, 06000 Bejaia, Algérie. \\ ${ }^{1}$ rachid_boukecha@yahoo.fr, ${ }^{2}$ mouna.yahiaoui89@gmail.com
}

Abstract. In this paper we are intersted in studying the existence of a first integral and to the curves which are formed by the trajectories of the two-dimensional differential systems of the form

$$
\left\{\begin{array}{l}
x^{\prime}=P(x, y)+x\left(\lambda x \exp \left(\frac{M(x, y)}{N(x, y)}\right)+\beta y \exp \left(\frac{R(x, y)}{S(x, y)}\right)\right) \\
y^{\prime}=Q(x, y)+y\left(\lambda x \exp \left(\frac{M(x, y)}{N(x, y)}\right)+\beta y \exp \left(\frac{R(x, y)}{S(x, y)}\right)\right)
\end{array}\right.
$$

where $P(x, y), Q(x, y), M(x, y), N(x, y), R(x, y), S(x, y)$ are homogeneous polynomials of degree $a, a, b, b, c, c$ respectively and $\lambda, \beta \in \mathbb{R}$. Concrete examples exhibiting the applicability of our result are introduced.

Keywords: Differential system, First integral, Curves.

2010 MSC No: 34C05, 34C07, 37C27, 37K10.

\section{Introduction}

We consider two-dimensional autonomous systems of differential equations of the form

$$
\left\{\begin{array}{l}
x^{\prime}=\frac{d x}{d t}=F(x(t), y(t)), \\
y^{\prime}=\frac{d y}{d t}=G(x(t), y(t)),
\end{array}\right.
$$

where $F(x, y)$ and $G(x, y)$ are reals functions. In the qualitative theory of planar dynamical systems [1,9, 10, 16], one of the most important topics is related to the second part of the unsolved Hilbert 16th problem [15]. There is a huge literature about limit cycles, most of them deal essentially with their detection, their number and their stability and rare are papers concerned by giving them explicitly $[2,4,7,13]$. There exist three main open problems in the qualitative theory of real planar differential systems, the distinction between a centre and a focus, the determination of the number of limit cycles and their distribution, and the determination of its integrability. The importance for searching first integrals of a given system was already noted by Poincaré in his discussion on a method to obtain polynomial or rational first integrals. One of the classical tools in the classification of all trajectories of a dynamical system is to find first integrals. For more details about first integral see for instance $[3,5,8,11,12,14]$, see the

\footnotetext{
${ }^{*}$ Corresponding author. Rachid Boukoucha ${ }^{1}$ rachid_boukecha@yahoo.fr
} 
references quoted in those articles. We recall that in the phase plane, a limit cycle of system (1) is an isolated periodic orbit in the set of all periodic orbits of system (1)

System (1) is integrable on an open set $\Omega$ of $\mathbb{R}^{2}$ if there exists a non constant $C^{1}$ function $H: \Omega \rightarrow \mathbb{R}$, called a first integral of the system on $\Omega$, which is constant on the trajectories of the system (1) contained in $\Omega$, i.e. if

$$
\frac{d H(x, y)}{d t}=\frac{\partial H(x, y)}{\partial x} F(x, y)+\frac{\partial H(x, y)}{\partial y} G(x, y) \equiv 0 \text { in the points of } \Omega .
$$

Moreover, $H=h$ is the general solution of this equation, where $h$ is an arbitrary constant. It is well known that for differential systems defined on the plane $\mathbb{R}^{2}$ the existence of a first integral determines their phase portrait [6].

In this paper we are intersted in studying the existence of a first integral and to the curves which are formed by the trajectories of the two-dimensional differential systems of the form

$$
\left\{\begin{array}{l}
x^{\prime}=P(x, y)+x\left(\lambda x \exp \left(\frac{M(x, y)}{N(x, y)}\right)+\beta y \exp \left(\frac{R(x, y)}{S(x, y)}\right)\right), \\
y^{\prime}=Q(x, y)+y\left(\lambda x \exp \left(\frac{M(x, y)}{N(x, y)}\right)+\beta y \exp \left(\frac{R(x, y)}{S(x, y)}\right)\right),
\end{array}\right.
$$

where $P(x, y), Q(x, y), M(x, y), N(x, y), R(x, y), S(x, y)$ are homogeneous polynomials of degree $a, a, b, b, c, c$ respectively and $\lambda, \beta \in \mathbb{R}$.

We define the trigonometric functions

$$
\begin{aligned}
& f_{1}(\theta)=\lambda(\cos \theta) \exp \left(\frac{M(\cos \theta, \sin \theta)}{N(\cos \theta, \sin \theta)}\right)+\beta(\sin \theta) \exp \left(\frac{R(\cos \theta, \sin \theta)}{S(\cos \theta, \sin \theta)},\right. \\
& f_{2}(\theta)=P(\cos \theta, \sin \theta) \cos \theta+Q(\cos \theta, \sin \theta) \sin \theta, \\
& f_{3}(\theta)=(\cos \theta) Q(\cos \theta, \sin \theta)-(\sin \theta) P(\cos \theta, \sin \theta) .
\end{aligned}
$$

\section{Main result}

Our main result on the integrability of differential system (2) is the following :

Theorem 1. Consider a differential system (2), then the following statements hold.

(1) If $f_{3}(\theta) N(\cos \theta, \sin \theta) S(\cos \theta, \sin \theta) \neq 0$ and $a \neq 2$, then system (2) has the first integral

$$
\begin{aligned}
H(x, y)= & \left(x^{2}+y^{2}\right)^{\frac{a-2}{2}} \exp \left((2-a) \int_{0}^{\arctan \frac{y}{x}} A(\omega) d \omega\right)- \\
& (a-2) \int_{0}^{\arctan \frac{y}{x}} \exp \left((2-a) \int_{0}^{w} A(\omega) d \omega\right) B(w) d w,
\end{aligned}
$$

where $A(\theta)=\frac{f_{2}(\theta)}{f_{3}(\theta)}, B(\theta)=\frac{f_{1}(\theta)}{f_{3}(\theta)}$, and the curves which are formed by the trajectories of the differential system (2), in cartesian coordinates are written as

$$
x^{2}+y^{2}=\left(\begin{array}{c}
h \exp \left((a-2) \int_{0}^{\arctan \frac{y}{x}} A(\omega) d \omega\right)+ \\
(a-2) \exp \left((a-2) \int_{0}^{\arctan \frac{y}{x}} A(\omega) d \omega\right) \\
\int_{0}^{\arctan \frac{y}{x}} \exp \left((2-a) \int_{0}^{w} A(\omega) d \omega\right) B(w) d w
\end{array}\right)^{\frac{2}{a-2}}
$$

where $h \in \mathbb{R}$.

(2) If $f_{3}(\theta) N(\cos \theta, \sin \theta) S(\cos \theta, \sin \theta) \neq 0$ and $a=2$, then system (2) has the first integral

$$
H(x, y)=\left(x^{2}+y^{2}\right)^{\frac{1}{2}} \exp \left(-\int_{0}^{\arctan \frac{y}{x}}(A(\omega)+B(\omega)) d \omega\right),
$$

and the curves which are formed by the trajectories of the differential system (2), in cartesian coordinates are written as

where $h \in \mathbb{R}$

$$
\left(x^{2}+y^{2}\right)^{\frac{1}{2}}-h \exp \left(\int_{0}^{\arctan \frac{y}{x}}(A(\omega)+B(\omega)) d \omega\right)=0,
$$

(3) If $f_{3}(\theta)=0$ for all $\theta \in \mathbb{R}$, then system (2) has the first integral $H=\frac{y}{x}$, and the curves which are formed by the trajectories of the differential system (2), in cartesian coordinates are written as $y-h x=0$, where $h \in \mathbb{R}$. 
Proof. In order to prove our results we write the polynomial differential system $(2)$ in polar coordinates $(r, \theta)$, defined by $x=r \cos \theta$ and $y=r \sin \theta$, then system (2) becomes

$$
\left\{\begin{array}{l}
r^{\prime}=f_{1}(\theta) r^{2}+f_{2}(\theta) r^{a} \\
\theta^{\prime}=f_{3}(\theta) r^{a-1}
\end{array}\right.
$$

where the trigonometric functions $f_{1}(\theta), f_{2}(\theta), f_{3}(\theta)$ are given in introduction, $r^{\prime}=\frac{d r}{d t}$ and $\theta^{\prime}=\frac{d \theta}{d t}$.

If $f_{3}(\theta) N(\cos \theta, \sin \theta) S(\cos \theta, \sin \theta) \neq 0$ and $a \neq 2$.

Taking as independent variable the coordinate $\theta$, this differential system (3) writes

$$
\frac{d r}{d \theta}=A(\theta) r+B(\theta) r^{3-a}
$$

where $A(\theta)=\frac{f_{2}(\theta)}{f_{3}(\theta)}$ and $B(\theta)=\frac{f_{1}(\theta)}{f_{3}(\theta)}$, which is a Bernoulli equation.

By introducing the standard change of variables $\rho=r^{a-2}$ we obtain the linear equation

$$
\frac{d \rho}{d \theta}=(a-2)(A(\theta) \rho+B(\theta)) .
$$

The general solution of linear equation (5) is

$$
\begin{aligned}
\rho(\theta)= & \exp \left((a-2) \int_{0}^{\theta} A(\omega) d \omega\right) \\
& \left(\mu+(a-2) \int_{0}^{\theta} \exp \left((2-a) \int_{0}^{w} A(\omega) d \omega\right) B(w) d w\right),
\end{aligned}
$$

where $\mu \in \mathbb{R}$, which has the first integral

$$
\begin{aligned}
H(x, y)= & \left(x^{2}+y^{2}\right)^{\frac{a-2}{2}} \exp \left((2-a) \int_{0}^{\arctan \frac{y}{x}} A(\omega) d \omega\right)- \\
& (a-2) \int_{0}^{\arctan \frac{y}{x}} \exp \left((2-a) \int_{0}^{w} A(\omega) d \omega\right) B(w) d w .
\end{aligned}
$$

Let $\Gamma$ be a periodic orbit surrounding an equilibrium located in one of the open quadrants, and let $h_{\Gamma}=H(\Gamma)$.

The curves $H=h$ with $h \in \mathbb{R}$, which are formed by trajectories of the differential system (2), in cartesian coordinates are written as

$$
x^{2}+y^{2}=\left(\begin{array}{c}
h \exp \left((a-2) \int_{0}^{\arctan \frac{y}{x}} A(\omega) d \omega\right)+ \\
(a-2) \exp \left((a-2) \int_{0}^{\arctan \frac{y}{x}} A(\omega) d \omega\right) \\
\int_{0}^{\arctan \frac{y}{x}} \exp \left((2-a) \int_{0}^{w} A(\omega) d \omega\right) B(w) d w
\end{array}\right)^{\frac{2}{a-2}}
$$

where $h \in \mathbb{R}$.

Hence statement (1) of Theorem 1 is proved.

Suppose now that $f_{3}(\theta) N(\cos \theta, \sin \theta) S(\cos \theta, \sin \theta) \neq 0$ and $a=2$.

Taking as independent variable the coordinate $\theta$, this differential system (3) writes

$$
\frac{d r}{d \theta}=(A(\theta)+B(\theta)) r
$$

The general solution of equation (6) is

$$
r(\theta)=\mu \exp \left(\int_{0}^{\theta}(A(\omega)+B(\omega)) d \omega\right),
$$


where $\mu \in \mathbb{R}$, which has the first integral

$$
H(x, y)=\left(x^{2}+y^{2}\right)^{\frac{1}{2}} \exp \left(-\int_{0}^{\arctan \frac{y}{x}}(A(\omega)+B(\omega)) d \omega\right) .
$$

The curves $H=h$ with $h \in \mathbb{R}$, which are formed by trajectories of the differential system (2), in cartesian coordinates are written as

$$
\left(x^{2}+y^{2}\right)^{\frac{1}{2}}-h \exp \left(\int_{0}^{\arctan \frac{y}{x}}(A(\omega)+B(\omega)) d \omega\right)=0,
$$

where $h \in \mathbb{R}$.

Hence statement (2) of Theorem 1 is proved.

Assume now that $f_{3}(\theta)=0$ for all $\theta \in \mathbb{R}$, then from system (3) it follows that $\theta^{\prime}=0$. So the straight lines through the origin of coordinates of the differential system (2) are invariant by the flow of this system. Hence, $\frac{y}{x}$ is a first integral of the system, then curves which are formed by the trajectories of the differential system (2), in cartesian coordinates are written as $y-h x=0$, where $h \in \mathbb{R}$, since all straight lines through the origin are formed by trajectories.

This completes the proof of statement (3) of Theorem 1.

\section{$3 \quad$ Examples}

The following examples are given to illustrate our result

Example 1 If we take $\lambda=1, \beta=-2, P(x, y)=2 x-3 y, Q(x, y)=3 x+2 y, M(x, y)=x^{2}+2 y^{2}$, $N(x, y)=x^{2}+y^{2}, R(x, y)=x^{4}+3 x^{2} y^{2}+y^{4}$ and $S(x, y)=x^{4}+2 x^{2} y^{2}+y^{4}$, then system (2) reads

$$
\left\{\begin{array}{l}
x^{\prime}=2 x-3 y+x\left(x \exp \left(\frac{x^{2}+2 y^{2}}{x^{2}+y^{2}}\right)-2 y \exp \left(\frac{x^{4}+3 x^{2} y^{2}+y^{4}}{x^{4}+2 x^{2} y^{2}+y^{4}}\right)\right), \\
y^{\prime}=3 x+2 y+y\left(x \exp \left(\frac{x^{2}+2 y^{2}}{x^{2}+y^{2}}\right)-2 y \exp \left(\frac{x^{4}+3 x^{2} y^{2}+y^{4}}{x^{4}+2 x^{2} y^{2}+y^{4}}\right)\right),
\end{array}\right.
$$

the differential system $(7)$ in polar coordinates $(r, \theta)$ becomes

$$
\left\{\begin{array}{l}
r^{\prime}=\left((\cos \theta) \exp \left(1+\sin ^{2} \theta\right)-2(\sin \theta) \exp \left(\frac{9}{8}-\frac{1}{8} \cos 4 \theta\right)\right) r^{2}+2 r, \\
\theta^{\prime}=3,
\end{array}\right.
$$

here $f_{1}(\theta)=(\cos \theta) \exp \left(1+\sin ^{2} \theta\right)-2(\sin \theta) \exp \left(\frac{9}{8}-\frac{1}{8} \cos 4 \theta\right), f_{2}(\theta)=2$ and $f_{3}(\theta)=3$, it is the case $(1)$ of the Theorem 1 .

The differential system (7) has the first integral

$$
H(x, y)=\left(x^{2}+y^{2}\right)^{\frac{-1}{2}} \exp \left(\frac{2}{3} \arctan \frac{y}{x}\right)-\int_{0}^{\arctan \frac{y}{x}} \exp \left(\frac{2}{3} w\right) B(w) d w,
$$

where $B(w)=\frac{(\cos w) \exp \left(1+\sin ^{2} w\right)-2(\sin w) \exp \left(\frac{9}{8}-\frac{1}{8} \cos 4 w\right)}{3}$.

The curves $H=h$ with $h \in \mathbb{R}$, which are formed by trajectories of the differential system (7), in cartesian coordinates are written as

$$
x^{2}+y^{2}=\left[\left(h+\int_{0}^{\arctan \frac{y}{x}} \exp \left(\frac{2}{3} w\right) B(w) d w\right) \exp \left(\frac{-2}{3} \arctan \frac{y}{x}\right)\right]^{-2},
$$

where $h \in \mathbb{R}$.

Example 2 If we take $\lambda=1, \beta=-2, P(x, y)=5 x^{2}+2 x y, Q(x, y)=-2 x y+5 y^{2}, M(x, y)=x^{2}+2 y^{2}$, $N(x, y)=x^{2}+y^{2}, R(x, y)=y$ and $S(x, y)=x$, then system (2) reads

$$
\left\{\begin{array}{l}
x^{\prime}=5 x^{2}+2 x y+x\left(x \exp \left(\frac{x^{2}+2 y^{2}}{x^{2}+y^{2}}\right)-2 y \exp \left(\frac{y}{x}\right)\right), \\
y^{\prime}=-2 x y+5 y^{2}+y\left(x \exp \left(\frac{x^{2}+2 y^{2}}{x^{2}+y^{2}}\right)-2 y \exp \left(\frac{y}{x}\right)\right),
\end{array}\right.
$$


the differential system (8) in polar coordinates $(r, \theta)$ becomes

$$
\left\{\begin{array}{l}
r^{\prime}=\left(7 \cos ^{3} \theta+3 \sin ^{3} \theta-2(\sin \theta)(-1+\exp \tan \theta)+(\cos \theta)\left(-2+\exp \left(1+\sin ^{2} \theta\right)\right)\right) r^{2}, \\
\theta^{\prime}=\left(3 \cos \theta \sin ^{2} \theta-7 \cos ^{2} \theta \sin \theta\right) r,
\end{array}\right.
$$

then the differential system (8) has the first integral

$$
H(x, y)=\left(x^{2}+y^{2}\right)^{\frac{1}{2}} \exp \left(-\int_{0}^{\arctan \frac{y}{x}}(A(\omega)+B(\omega)) d \omega\right)
$$

where $A(\omega)+B(\omega)=\frac{7 \cos ^{3} \omega+3 \sin ^{3} \omega-2(\sin \omega)(-1+\exp \tan \omega)+(\cos \omega)\left(-2+\exp \left(1+\sin ^{2} \omega\right)\right)}{3 \cos \omega \sin ^{2} \omega-7 \cos ^{2} \omega \sin \omega}$, it is the case (2) of the Theorem 1 ,

The curves $H=h$ with $h \in \mathbb{R}$, which are formed by trajectories of the differential system (8), in cartesian coordinates are written as

$$
\left(x^{2}+y^{2}\right)^{\frac{1}{2}}-h \exp \left(\int_{0}^{\arctan \frac{y}{x}}(A(\omega)+B(\omega)) d \omega\right)=0,
$$

where $h \in \mathbb{R}$.

Example 3 If we take $\lambda=1, \beta=-2, P(x, y)=x^{3}+x y^{2}, Q(x, y)=y^{3}+y x^{2}, M(x, y)=x^{2}+2 y^{2}$, $N(x, y)=x^{2}+y^{2}, R(x, y)=x^{4}+3 x^{2} y^{2}+y^{4}$ and $S(x, y)=x^{4}+2 x^{2} y^{2}+y^{4}$, then system (2) reads

$$
\left\{\begin{array}{l}
x^{\prime}=x^{3}+x y^{2}+x\left(x \exp \left(\frac{x^{2}+2 y^{2}}{x^{2}+y^{2}}\right)-2 y \exp \left(\frac{x^{4}+3 x^{2} y^{2}+y^{4}}{x^{4}+2 x^{2} y^{2}+y^{4}}\right)\right), \\
y^{\prime}=y^{3}+y x^{2}+y\left(x \exp \left(\frac{x^{2}+2 y^{2}}{x^{2}+y^{2}}\right)-2 y \exp \left(\frac{x^{4}+3 x^{2} y^{2}+y^{4}}{x^{4}+2 x^{2} y^{2}+y^{4}}\right)\right),
\end{array}\right.
$$

the differential system (9) in polar coordinates $(r, \theta)$ becomes

$$
\left\{\begin{array}{l}
r^{\prime}=r^{\prime}=r^{3}+\left((\cos \theta) \exp \left(1+\sin ^{2} \theta\right)-2(\sin \theta) \exp \left(\frac{9}{8}-\frac{1}{8} \cos 4 \theta\right)\right) r^{2}, \\
\theta^{\prime}=0
\end{array}\right.
$$

it is the case (3) of the Theorem 1, then from system (10) it follows that $\theta^{\prime}=0$. Hence, $\frac{y}{x}$ is a first integral of the system, then curves which are formed by the trajectories of the differential system (9), in Cartesian coordinates are written as $y-h x=0$, where $h \in \mathbb{R}$, since all straight lines through the origin are formed by trajectories.

\section{Conclusion}

The elementary method used in this paper seems to be fruitful to investigate planar differential systems of ODEs in order to obtain explicit expression for a first integral and characterizes its trajectories, this is a one of the classical tools in the classification of all trajectories of dynamical systems.

\section{References}

[1] A. A. Andronov, E. A. Leontovich, I. I. Gordon, and A. L. Maier, Qualitative Theory of Second-Order Dynamical Systems, Wiley, New York, 1973. https://doi.org/10.31559/glm2016.3.2.3

[2] A. Bendjeddou, R. Boukoucha, Explicit limit cycles of a cubic polynomial differential systems, Stud. Univ. Babes-Bolyai Math, 2016, vol. 61, no. 1, pp. 77-85.

[3] R. Boukoucha, On the Dynamics of a Class of Kolmogorov Systems, Journal of Siberian Federal University. Mathematics \& Physics 2016, vol. 9, no. 1, pp. 11-16. https://doi.org/10.4236/ajcm.2013.33026

[4] R. Boukoucha, Explicit limit cycles of a family of polynomial differential systems, Electronic Journal of Differential Equations, 2017, vol. 2017, no. 217, pp. 1-7. https://doi.org/10.1016/j.amc.2005.07.072

[5] R. Boukoucha, A. Bendjeddou, On the dynamics of a class of rational Kolmogorov systems, Journal of Nonlinear Mathematical Physics, 2016, vol. 23, no. 1, pp. 21-27. 
[6] L. Cairó, J. Llibre, Phase portraits of cubic polynomial vector fields of Lotka-Volterra type having a rational first integral of degree 2, J. Phys, 2007, A 40, pp. 6329-6348.

[7] M. Carbonell, B. Coll and J. Llibre, Limit cycles of polynomial systems with homogeneous nonlinearities, J. of Math. Anal. and Appl, 1989, vol. 142, pp. 573-590.

[8] J. Chavarriga, H. Giacomini, J. Giné, J. Llibre, Darboux integrability and the inverse integrating factor, J. Differential Equations, 2003, vol. 194, pp. 116-139.

[9] C. Christopher, C. Li, S. Yakovenko, Advanced Course On Limit Cycles of Differential Equations, Centre de Recerca Matemàtica Bellaterra (Spain), (2006).

[10] F. Dumortier, J. Llibre and J. Artés, Qualitative Theory of Planar Differential Systems, (Universitex) Berlin, Springer, (2006).

[11] P. Gao, Hamiltonian structure and first integrals for the Lotka-Volterra systems, Phys. Lett, 2000, A 273, pp. 85-96.

[12] J. Giné. Polynomial first integrals via the Poincaré series, J. Comput. Appl. Math, 2005, vol. 184 , pp. $428-441$.

[13] J. Giné, M. Grau, Coexistence of algebraic and non-algebraic limit cycles, explicitly given, using Riccati equations. Nonlinearity, 2006, vol. 19, pp. 1939-1950.

[14] N. A. Korol, The integral curves of a certain differential equation, (in Russian), Minsk. Gos. Ped. Inst. Minsk, 1973, pp. 47-51.

[15] D. Hilbert, Mathematische Probleme, Lecture, Second Internat, English transl, Bull. Amer. Math. Soc, 1902, vol. 8, pp. 437-479.

[16] L. Perko, Differential equations and dynamical systems, Third edition. Texts in Applied Mathematics, 7. Springer-Verlag, New York, 2001. 